\title{
Kein Sport vor dem Rheuma-Ultraschall
}

Wer sich bei Verdacht auf entzündliches Rheuma einem Gelenkultraschall unterzieht, sollte vorher auf Sport verzichten. Denn körperliche Anstrengung am Vortag kann die Diagnose von Gelenkrheuma erschweren.

Ultraschalluntersuchungen spielen sowohl bei der Diagnostik als auch bei der Behandlung der Rheumatoiden Arthritis eine entscheidende Rolle [1]: Sie ermöglichen es, frühzeitig entzündliche Prozesse aufzudecken, und sie geben Hinweise darauf, welche Therapie die richtige ist. „Um Unsicherheiten auszuschließen, sollte man vor einem Gelenkultraschall ein paar Ruhetage einlegen", so Prof. Dr. Marina Backhaus, Chefärztin der Abteilung Innere Medizin - Rheumatologie und Klinische Immunologie an der Park-Klinik Weißensee in Berlin. Die Expertin der DEGUM (Deutsche Gesellschaft für UItraschall in der Medizin) verweist auf eine Studie aus Spanien: Die Wissenschaftler hatten bei Probanden, die sich am Vortag körperlich betätigten, deutlich häufiger Hinweise auf eine Gelenkinnenhautentzündung registriert als bei Kontrollpersonen. „Eine solche Synovitis kann Folge einer rheumatischen
Erkrankung sein, muss es aber nicht", erklärt Backhaus. Manchmal sei die Ursache - wie bei einer Sehnenscheidenentzündung - nur eine Überanstrengung oder Überreizung, so die Expertin.

Ultraschalluntersuchungen eignen sich sehr gut zur Frühdiagnostik rheumatischer Erkrankungen. Auf dem Bildschirm lassen sich selbst kleinste Defekte in Knorpel und Knochen der Hand- und Fingergelenke erkennen. Erfahrene Untersucher können dem Ultraschallbild zudem zuverlässige Hinweise auf entzündliche Aktivitäten entnehmen. Das ist vor allem bei Patienten mit einer bereits diagnostizierten RA wichtig. „Nicht immer schätzen die Patienten die Krankheitsaktivität richtig ein", erläutert Backhaus.

Neben dem gewöhnlichen B-Mode Ultraschall setzen Rheumatologen und Orthopäden dabei auch den Doppler-Ultraschall ein. Mit dieser Methode können die Ärzte vermehrte Gefäßbil- dungen in der Gelenkschleimhaut nachweisen und so einen sich anbahnenden rheumatischen Schub vorhersagen.

Insbesondere bei Patienten, bei denen Beschwerden wie Morgensteifigkeit, Müdigkeit oder Schmerzen dauerhaft oder vorübergehend abgeschwächt sind, kann dies wertvolle Erkenntnisse liefern. „Die Ergebnisse haben mitunter großen Einfluss auf die Wahl der Therapie", erklärt Backhaus. Die Expertin weiter: „Für die Sonografie sind Qualifikation und Erfahrung des Untersuchers von entscheidender Bedeutung."

\section{Literatur \\ 1. Ohrndorf S, Backhaus M (2015) Gelenk-UI- traschall bei rheumatoider Arthritis. Dtsch Med Wochenschr 140:1223-1226. doi:10.10 55/s-0041-103783}

rheuma plus $2016 \cdot 15: 31$

DOI 10.1007/s12688-016-0068-0

Online publiziert: 31. Mai 2016

C) Springer-Verlag Wien 2016
Quelle: Pressemitteilung der Deutschen Gesellschaft für Ultraschall in der Medizin (DEGUM)

\section{Neuer Ansatz für die Behandlung von Lupus}

Eine Forschungsgruppe der MedUni Wien hat im Tiermodell einen neuen Ansatzpunkt für eine mögliche Therapie des Systemischen Lupus Erythematosus (SLE) entdeckt. Die Blockade von Interleukin-6 (IL-6) verhindert dessen Andocken an die Rezeptoren und damit die Entstehung von Entzündungen. Die Arbeit wurde im Journal „Experimental Dermatology“ publiziert.

Der Forschergruppe um Prof. Dr. Peter Birner (MedUni Wien) und Prof. Dr. Lukas Kenner (MedUni Wien, Veterinärmedizinische Universität Wien und Ludwig Boltzmann Institut für Krebsforschung) sowie Wissenschaftlern aus Graz, Deutschland und Japan gelang erstmals der experimentelle Nachweis, dass eine
Blockade von IL-6 bei SLE im Tiermodell einen signifikanten Heilungseffekt haben kann.

Bereits in einer früheren Publikation konnte die Arbeitsgruppe um Kenner zeigen, dass bei Autoimmunerkrankungen wie SLE die Produktion von Interleukin 6 (IL-6) aus Keratinozyten der Haut eine Schlüsselrolle bei der Ent- stehung und Progression der Erkrankung spielt. Dafür wurde ein transgenes Mausmodell verwendet, bei dem der AP-1 Transkriptionsfaktor JunB in der Epidermis ausgeschalten war. Dabei zeigte sich, dass in der Haut die Expression von JunB die Produktion von IL-6 inhibiert. Der Verlust von JunB in der Haut führte dann zu einer sehr ho- 